\title{
Sex differences in salivary cortisol reactivity to the Trier Social Stress Test (TSST): A meta-analysis
}

\author{
Jenny J.W. Liu ${ }^{\mathrm{a}, \mathrm{b}}$, Natalie Ein ${ }^{\mathrm{a}, \mathrm{b}}$, Katlyn Peck ${ }^{\mathrm{a}, \mathrm{b}}$, Vivian Huang ${ }^{\mathrm{a}, \mathrm{b}}$, Jens C. Pruessner ${ }^{\mathrm{c}}$, \\ Kristin Vickers ${ }^{\mathrm{a}, \mathrm{b}, *}$ \\ a Department of Psychology, Ryerson University, Toronto, Ontario, Canada \\ b Institute for Stress and Wellbeing Research, Ryerson University, Toronto, Ontario, Canada \\ ${ }^{c}$ Department of Psychology, McGill University, Montreal, Quebec, Canada
}

Keywords:

Sex

Salivary cortisol

Trier Social Stress Test (TSST)

\begin{abstract}
A B S T R A C T
Some, but not all studies using the Trier Social Stress Test (TSST) have demonstrated evidence in support of sex differences in salivary cortisol. The aim of the current meta-analysis is to examine sex differences in salivary cortisol following exposure to the TSST. We further explored the effects of modifications to the TSST protocol and procedural variations as potential moderators. We searched articles published from January, 1993 to February, 2016 in MedLine, PsychINFO, and ProQuest Theses and Dissertations. This meta-analysis is based on 34 studies, with a total sample size of 1350 individuals ( 640 women and 710 men). Using a random effects model, we found significant heterogeneity in salivary cortisol output across sexes, such that men were observed to have higher cortisol values at peak and recovery following the TSST compared to women. Modifications to the sampling trajectory of cortisol (i.e., duration of acclimation, peak sampling time, and duration of recovery) significantly moderated the heterogeneity across both sexes. Further, there are observed sex differences at various time points of the reactive cortisol following the TSST. Lastly, current results suggest that these sex differences can be, at least in part, attributed to variations in methodological considerations across studies. Future research could advance this line of inquiry by using other methods of analyses (e.g., area under the curve; AUC), in order to better understand the effects of methodological variations and their implications for research design.
\end{abstract}

\section{Introduction}

Sex differences in stress reactivity have increasingly received empirical attention (Juster et al., 2016). The Trier Social Stress Test (TSST) is a well-validated and widely used method to induce an acute stress response within a laboratory setting (Dickerson and Kemeny, 2004; Kirschbaum et al., 1993). The original protocol proposed by Kirschbaum et al. (1993), is a standardized procedure that reliably elicits moderate to large physiological and subjective stress responses across different age groups in both sexes (Kudielka et al., 2004).

The components of the standardized TSST include an anticipation period, a speech and a verbal mental arithmetic task. The testing procedure begins with participants gradually adjusting to the laboratory environment for $30 \mathrm{~min}$ in the first testing room (room A), known as the acclimation period. Participants are then guided to a second room (room B) where they are given specific instructions regarding the speech task, and are introduced to the panel of evaluators (confeder- ates). Subsequently, participants are then led back to room A, and are given $10 \mathrm{~min}$ to prepare their speech (e.g., mock job interview). Following the anticipatory period, participants return to room B and perform their speech ( $5 \mathrm{~min}$ ), followed by a verbal mental arithmetic task $(5 \mathrm{~min})$ in front of the panel of evaluators. To increase social evaluative threat, participants are informed that their performance will be video recorded and the evaluators are instructed to adopt a nonresponsive demeanor. Upon completion, participants are escorted back to room A for the duration of the recovery period (30-70 min depending on the target hormones being measured). When reproduced, components of the TSST may vary slightly across studies (e.g., length of each task, content of speech, etc.), which may affect variability in reactivity in the sampled population (Kirschbaum et al., 1993).

Cortisol is the final output of the hypothalamic pituitary adrenal (HPA) axis, the main hormonal stress system within the body and is commonly used as a biomarker of psychological stress (Hellhammer et al., 2009; Kirschbaum et al., 1993). Obtaining cortisol from saliva is

\footnotetext{
Corresponding author at: JOR 923, Ryerson University, 350 Victoria Street, Toronto, Ontario M5B 2K3, Canada.

E-mail address: kvickers@psych.ryerson.ca (K. Vickers).
} 
considered to be a less invasive procedure compared to other methods (e.g., serum), which likely contributes to the large popularity of the approach. Further, given that the biologically active fraction of cortisol is reflected in saliva, it can be a preferred measure relative to serum cortisol (Hellhammer et al., 2009; Kudielka et al., 2012).

The standardized protocol by Kirschbaum et al. (1993) recommends the collection of saliva in 10-30 min intervals throughout the experiment for subsequent analysis of cortisol. Typically, the baseline value of cortisol is collected $30 \mathrm{~min}$ following acclimation and prior to the start of the TSST. The duration of the acclimation period is necessary to ensure that participants have sufficient time to adapt to the laboratory environment. To observe peak reactivity of salivary cortisol, it is typically collected between 10 and 25 min post-TSST, whereas recovery collections extend up to $70 \mathrm{~min}$ post-TSST. Given the time-sensitive nature of cortisol reactivity, altering the time-course of cortisol collection may influence the cortisol values obtained (Del Giudice et al., 2011).

In addition to alterations in sampling time points, biological sex is a significant factor influencing cortisol responses to acute psychosocial stress and subsequent cortisol output (Kirschbaum et al., 1992). Consistent sex differences exist in cortisol output in response to acute stress (see Zimmer et al., 2003), with most studies reporting higher cortisol output in men following the TSST (reviewed in Kudielka and Kirschbaum, 2005; for non-significant difference see, for example, Kelly et al., 2008). Following exogenous cortisol administration, women tend to show higher levels of glucocorticoids compared to men (Gaffey et al., 2014). Further, men tend to exhibit a significantly higher anticipatory cortisol response to stress relative to women (Engert et al., 2013; Kirschbaum et al., 1992).

Finally, it has been postulated that women tend to respond to stress in more socially oriented ways (Taylor et al., 2014), such that they may be more susceptible to the social-evaluative components within the TSST. Past meta-analyses exploring sex differences have not provided conclusive support for specific sex differences in cortisol reactivity during experiments using TSST, and have yet to evaluate factors that may contribute to these differences (Dickerson and Kemeny, 2004; Otte et al., 2005). Thus, it is unclear whether components within the TSST can lead to varied responses across both sexes. Although gender and biological sex could both distinctively contribute to differences in salivary cortisol, it should be noted that the focus of this meta-analysis is exclusive to biological sex, whereas the discussion of gender as a construct is beyond the scope of the current study.

The goal of the current meta-analysis is to substantiate sex differences in salivary cortisol following exposure to the TSST. Importantly, an exploratory aim of our meta-analysis is to examine how modifications to the TSST (length of tasks, content of speech and presence of confederates) and procedural variations (length of acclimation, time of sampling peak, length of recovery, and the number of saliva samples) may influence salivary cortisol output across sexes.

\section{Method}

\subsection{Study selection}

Our searches were conducted using Medline, PsychINFO, and Proquest Theses and Dissertations databases. The search was completed on February 17, 2016, and incorporated articles that were published since the conception of TSST (September 1993; Kirschbaum et al., 1993) to February 2016. The search terms included "cortisol" AND "Trier Social Stress Test" OR "TSST". Articles that contained the abovenoted keywords were retained.

\subsection{Inclusion and exclusion criteria}

There were four inclusion criteria for the current meta-analysis: 1) healthy human adults between the ages of 18 and 65 to ensure sampling of normative cortisol levels; 2) participants who completed all portions of the TSST (i.e., anticipation, speech and mental arithmetic); 3) salivary cortisol as the sole sampling method; and 4) collection of a minimum of three salivary cortisol samples to capture the time course of reactive cortisol secretion following stress exposure.

Articles were excluded based on the following: (1) studies that employed clinical samples and/or pharmacological intervention, as either may alter cortisol reactivity compared to a healthy population; (2) studies with a focus on identifying genetic polymorphisms as they were often grouped based on allele types, rather than sex, and may serve as a confound; (3) studies with multiple stressors or multiple TSST exposures, as stress habituation and knowledge of additional exposures can affect cortisol output; and (4) studies that had $5 \mathrm{~min}$ or less before and after the TSST as this duration of time is not sufficient for acclimation and recovery before and after exposure to stress.

The literature search identified 1916 articles and theses. After removing duplicates, 1510 unique published and unpublished studies were identified. The relevance of every article was determined in two stages: title and abstract, and full article. After applying the inclusion and exclusion criteria, a total 1315 articles were excluded based on information found in the study's title and abstracts. If the necessary information was not provided in the titles and abstracts to make a confident decision, the article was retained for full-text review. Of the remaining 195 articles, full texts were retrieved and reviewed in their entirety to ensure the studies adhered to the inclusion and exclusion criteria. At this stage, 129 full text articles were excluded. A total of 66 articles were reviewed and retained. Of these articles, 22 studies contained data that could be extracted via in text or published figures. Web Plot Digitizer (Rohatgi, 2016) was used to extract the means and standard deviations of cortisol at each time point in study figures. The remaining 44 studies did not report the necessary information to allow for data extraction (i.e., mean cortisol values and standard deviations separated by sex) and required author contact to request missing information. A total of 12 authors provided useable data that were included in the analysis. Overall, a total of 34 studies with independent samples were included in the current analysis (see Fig. 1). Three authors (J.L., N.E., \& V.H.) evaluated the titles and abstracts independently. Final article selected based on full text review was done by J.L. and N.E. All discrepancies regarding final article selection was resolved through discussion; however, there were no disagreements in final article evaluations (i.e., inter-rater reliability was $100 \%$ ).

\subsection{Data extraction and coding}

Sample characteristics and study results were extracted, including: sex distribution of sampled population, age of participants, time points of cortisol sampling, and the means and standard deviations of each cortisol sample separated by sex. To ensure equivalence among units of measurements across studies, we converted all units to nanomoles per liter $(\mathrm{nmol} / \mathrm{L})$. The general TSST characteristics were extracted as possible moderators. If available, the following information regarding any modifications to the original, standardized TSST procedures were included: length of each task, speech content, and presence of confederates. These were further coded as yes if different from the standardized protocol, or no, if they did not deviate from the standardized TSST protocol (Kirschbaum et al., 1993).

Of the extracted study characteristics, the duration of the experiment varied largely between studies. In particular, the length of the TSST and cortisol collection time points were often inconsistent across study methodologies. To address this, we standardized the timeline of the experimental procedure to normalize the differences in TSST duration by measuring time, in minutes, relative to the TSST. We coded the entirety of the TSST protocol, starting with the anticipation period as 0 ; the length of the baseline period prior to anticipation was coded as negative in minutes, in relation to the TSST; and the length of time following the arithmetic component of the TSST was coded as 


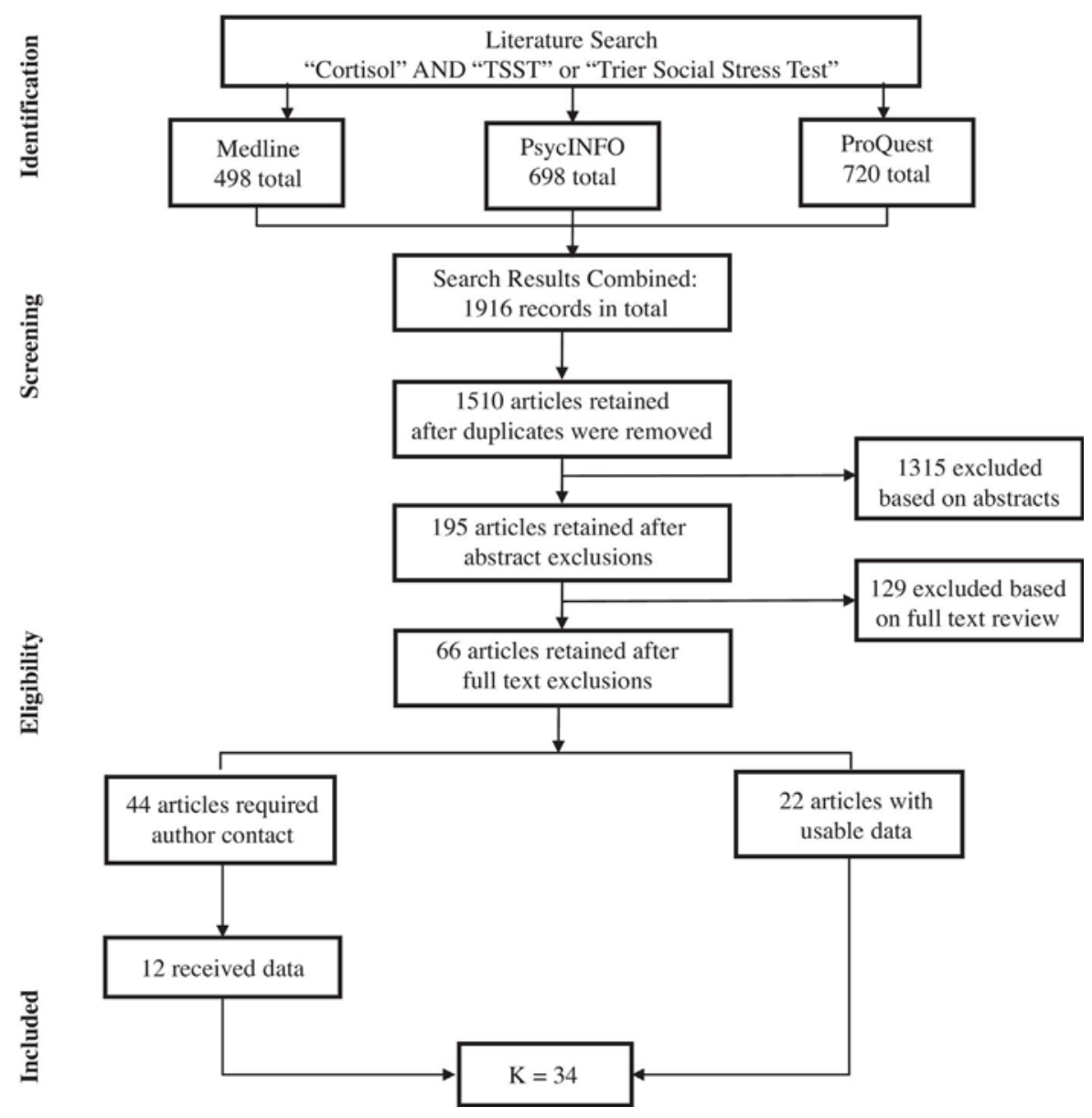

Fig. 1. Preferred reporting items for systematic reviews and meta-analysis (PRISMA) flowchart of the procedure used in article selection.

positive in minutes, in relation to the TSST. Additionally, we standardized the timeline of the cortisol collection to normalize the range as representative of that of reactive cortisol. We coded the time points into three cortisol trajectory phases: baseline, peak and recovery. Baseline was determined by selecting the sampling time point toward the end of a period of acclimation, and closest to the start of the TSST (time closest to $-1 \mathrm{~min}$; Kirschbaum et al., 1993). Peak was determined by selecting the time point with the highest cortisol value that ranged between 1 and 30 min post-TSST. Finally, recovery was established by selecting the time point closest to $60 \mathrm{~min}$ following the termination of the TSST, as this ensures adequate time for recovery.

Lastly, we also coded for additional study characteristics that may have influenced salivary cortisol output, including sampling time of the day (Putnam et al., 2009), menstrual cycle (Villada et al., 2014a,b), and oral contraceptive use (OC; Roche et al., 2013). With regards to sampling time, 30 studies reported information on time of day in cortisol sampling. Of these studies, 29 sampled cortisol in the afternoon and evening, between 12:00 pm to 19:30 pm, while one study sampled during the morning. The remaining four studies did not provide this information. As such, sampling time of the day could not be used as a moderator. Of the 26 studies that had female samples, six studies contained information on menstrual cycle, and were coded as a binary moderator, whereby studies either controlled for menstrual cycle (sampling within late luteal or follicular phases), or they did not. Additionally, OC use was coded as a binary moderator, whereby studies either controlled for oral contraceptive use by sampling only women on OC, or studies that only sampled women that were not on OC. Of these studies, seven contained women not on OC, while five used samples on OC.

\subsection{Data analytic plan}

All analyses were conducted using Comprehensive Meta-Analysis software (CMA) version 3.0 software (Borenstein et al., 2014). The following information was inserted into CMA: sex, sample size, mean and standard deviation of cortisol at baseline, peak and recovery. Sex was entered as a subgroup within each study (men and women), while time points were entered as separate outcomes within the study (baseline, peak, and recovery). For the main analysis of sex, we used $Q$ statistics to assess for homogeneity of group means across various study outcomes (baseline, peak, and recovery). Using a random effects model, sex differences are reported as mean difference.

An additional goal of our meta-analysis was to examine the impact of modifications to the TSST protocol on cortisol reactivity. We therefore conducted a moderator analysis on the effects of modification on salivary cortisol outputs. We also assessed for the impact of additional study procedural variations on cortisol output as possible moderators, which included the number of cortisol samples, length of acclimation period, time of peak cortisol value, and length of recovery period. A meta-regression with random effects model with moment method examined the impact of these moderators on overall homogeneity. After identifying significant moderators through a meta-regression, J.L. and N.E. then categorically coded moderators based on whether they deviated from the original study design of a TSST (Kirschbaum et al., 1993). Finally, $Q$ statistics were used to examine the specific moderator's effects on homogeneity of both sexes' salivary cortisol output, using a random effects model.

Using Duval and Tweedie's (2000) trim-and-fill procedure, we examined potential publication bias. Using this method, we examined the plotted means of each study against a funnel plot generated of 
imputed means (precision of study). The visual plot should represent a funnel if no publication bias is present. Further, we used a fail-safe analysis to examine the number of unpublished studies required to nullify the meta-analytic findings.

\section{Results}

\subsection{Study characteristics}

Overall, a total of 34 studies were included for the purpose of our meta-analysis. Across studies, the sample size included 1350 individuals ( 640 women and 710 men). Of the 34 studies, 20 studies had both male and female participants, seven studies had only women, and seven studies had only men. The age range was between 18 and 61 years old. The average number of salivary cortisol samples collected throughout studies was $5(S D=2.25$; range $=3-13)$. The length of acclimation had an average of $23 \mathrm{~min}(S D=12.35$; range $=10-60)$. The time of peak cortisol post-TSST had an average of $13 \mathrm{~min}(S D=7.68$; range $=1-32.50$ ). The length of recovery had an average of $46 \mathrm{~min}$ $(S D=20.18$; range $=15-90)$. Fifteen studies did not modify the TSST from the standard protocol, compared to 19 studies that modified the TSST to some extent (e.g., shortened length of tasks, change of speech content, and absence of confederates). Characteristics of studies that were included in the current meta-analyses are shown in Table 1.

\subsection{Sex differences in salivary cortisol across study outcomes}

Across studies, we examined the relationship between sex and salivary cortisol at baseline. The mean difference between women CI $[6.15,8.35]$ and men CI $[6.82,9.18]$ was $0.75\left(S E_{D}=0.05\right)$. Test of homogeneity revealed sex did not have a significant effect on salivary cortisol at baseline prior to TSST ( $Q=0.84, d f=1, p=.36$ ). Next, we examined the relationship between sex and salivary cortisol at peak in response to the TSST. The mean difference between women's CI [8.22, 11.05] and men's CI [13.04, 16.12] was $4.95\left(S E_{D}=0.31\right)$, whereby men had higher values compared to women. Test of homogeneity revealed sex accounted for a significant amount of heterogeneity in salivary cortisol at peak $(Q=21.50, d f=1, p<.001)$. Finally, we examined the relationship between sex and salivary cortisol during recovery. The mean difference between women's CI [6.43, 8.51] and men's CI $[8.03,10.24]$ was $1.66\left(S E_{D}=0.09\right)$, whereby men had higher values compared to women. Test of homogeneity revealed sex accounted for a significant amount of heterogeneity in salivary cortisol during recovery $(Q=4.61, d f=1, p=.03)$. These differences are shown in Figs. 2 and 3.

\subsection{Effects of modifications and procedural variations on salivary cortisol}

Of the 34 studies, 19 studies modified the standardized TSST protocol. Therefore, we examined whether modification to the TSST accounted for any heterogeneity across salivary cortisol. No differences in salivary cortisol output were observed between studies that adhered to, or deviated from, the standardized TSST protocol ( $p$ values ranged from $p=.30$ to .88 ).

A meta-regression using a random effects model with moment method was conducted to determine whether procedural variations moderated the magnitude of effect sizes in salivary cortisol output. Using a backwards step method, procedural variations (acclimation, peak, recovery and saliva samples) were entered in a linear regression model. The $Q$-statistics determined that at least some of the procedural variations significantly related to the effect size $(Q=33.02, d f=3$, $p<.001)$. Together, acclimation, peak and recovery accounted for $36 \%$ of the variance in sex differences found in salivary cortisol. Results showed that acclimation $(p=.04)$, peak $(p<.001)$, and recovery $(p<.001)$ accounted for significant differences in variance in salivary cortisol. The number of salivary cortisol samples taken did not account for any significant variance $(b=0.20$, CI $[-0.13,0.53], Z=1.17$, $p=.24$ ). The coefficients for model change and individual procedural variations are shown in Table 2.

Based on significant model changes in variance accounted for through procedural variations, we examined the differences in these three variables based on the original study protocol as proposed by Kirschbaum et al. (1993). For the significant coefficient found on length of acclimation ( $b=0.05$, CI [0.00, 0.09], $Z=2.03, p=.04)$, we compared the recommended length of acclimation $(\geq 30 \mathrm{~min}$ ) with studies that did not meet this requirement ( $\leq 29 \mathrm{~min}$ ), and categorically tested for homogeneity across sex and cortisol outcomes. For studies that met the required length of acclimation, we found no significant heterogeneity across sex in cortisol output at any time points ( $p=.13-.85$ ). However, for studies that did not met the requirement, we found significant heterogeneity in salivary cortisol between sex at all time points (baseline, peak, and recovery; $p<.05$ ). Summary statistics for $Q$ values are shown in Table 3.

Based on significant model changes in variance accounted for through sampling time point at cortisol peak value $(b=0.19$, CI $[0.11,0.27], Z=4.65, p<.001)$, we compared the recommended time frame for cortisol peak (between 10 and $25 \mathrm{~min}$ ) against studies that sampled outside the mentioned time ( $\leq 9 \mathrm{~min}$ or $\geq 26 \mathrm{~min}$ ), and categorically tested for homogeneity across sex and cortisol outcomes. For studies that sampled peak within the recommended time frame, our results did not differ from the initial sex differences without moderators. However, for studies that sampled peak outside of the recommended time frame, we no longer detected significant heterogeneity across sex on salivary output at peak and recovery $(p=.37-.99)$. Summary statistics for $Q$ values are shown in Table 3 .

Based on significant model changes in variance accounted for through length of recovery $(b=-0.07$, CI $[-0.10,-0.04]$, $Z=-4.48, p<.001$ ), we compared the required length of recovery ( $\geq 70 \mathrm{~min}$ ) compared to studies that did not provide adequate length of recovery ( $\leq 69 \mathrm{~min}$ ). Of the studies that provided a standardized length of recovery, we no longer detected heterogeneity across sex in salivary cortisol at peak and recovery ( $p=.26$ to .44$)$. However, in studies that provided shorter recovery periods, our results did not differ from the initial sex differences observed in salivary cortisol. Summary statistics for $Q$ values are shown in Table 3.

\subsection{Menstrual cycle and oral contraceptive use (women only)}

We examined whether controlling for menstrual cycle had any effect on salivary cortisol output in women across different time points. Tests of homogeneity revealed that the effect of menstrual cycle on salivary cortisol in women were not significant at baseline $(Q=0.10, d f=1$, $p=.75)$, peak $(Q=0.22, d f=1, p=.64)$, or recovery $(Q=0.06$, $d f=1, p=.81$ ).

Next, we examined whether the use of OC had any effect on salivary cortisol output in women across different time points. At baseline, the mean difference between women not using OC CI $[6.24,9.56]$ and those using OC CI $[3.03,7.38]$ was $2.70\left(S E_{D}=0.36\right)$. Test of homogeneity revealed the effects of OC use on salivary cortisol in women approached significance at baseline $(Q=3.74, d f=1$, $p=.05)$. At peak, the mean difference between women not using OC CI $[9.44,12.66]$ and those using OC CI $[4.40,8.37]$ was 4.66 $\left(S E_{D}=0.62\right)$, whereby women not on OC were observed to have higher values compared to women on OC. Test of homogeneity revealed that OC use resulted in significant heterogeneity in salivary cortisol values in women during peak $(Q=12.83, d f=1, p<.001)$. Finally, during recovery, the mean difference between women not using OC CI $[5.48,9.54]$ and those using OC CI $[2.76,8.15]$ was $2.05\left(S E_{D}=0.27\right)$. Test of homogeneity revealed that $\mathrm{OC}$ use did not have a significant effect on salivary cortisol in women at recovery $(Q=1.42, d f=1$, $p=.23)$.

Given the significant differences in values within women as a result 


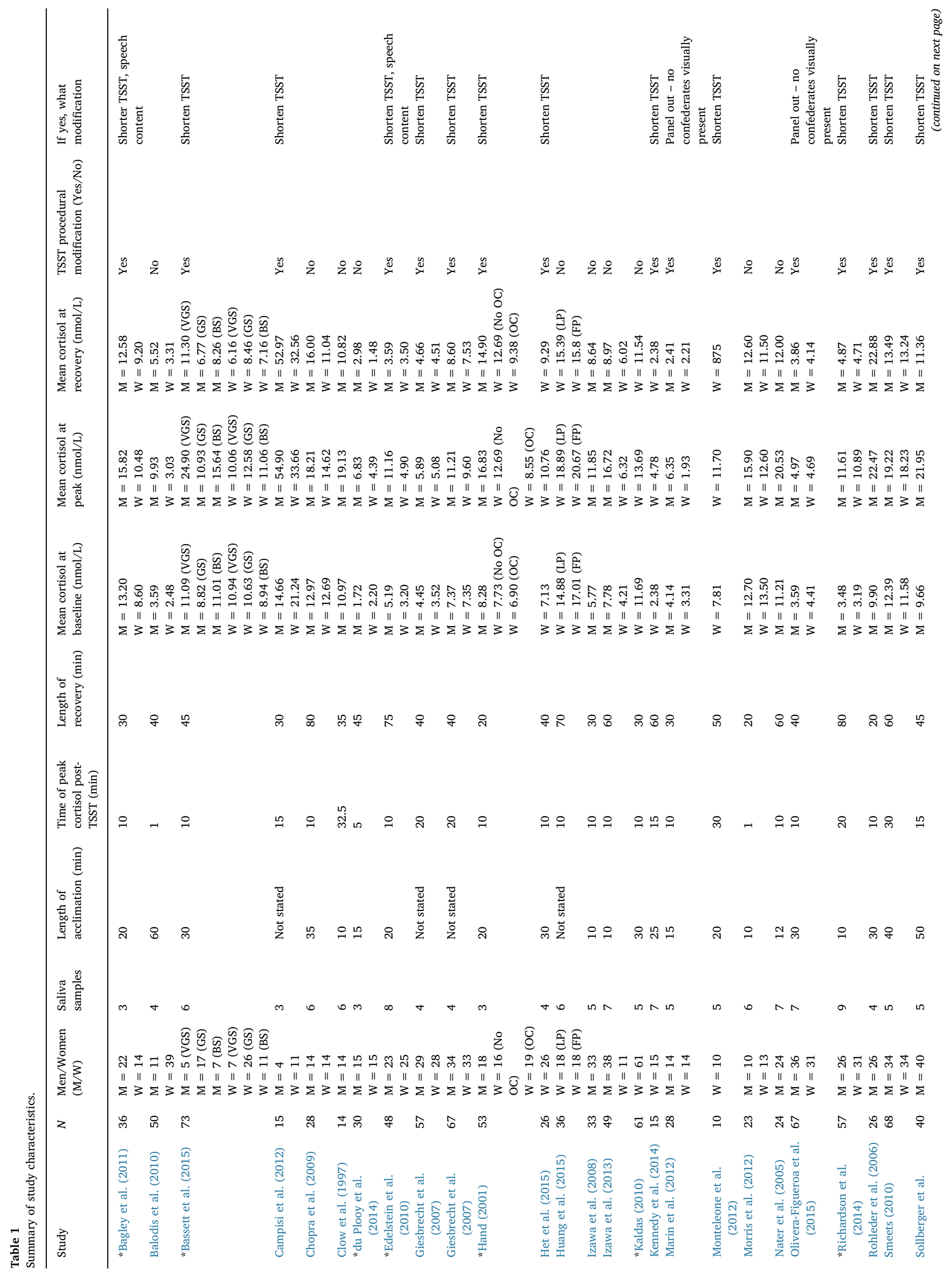




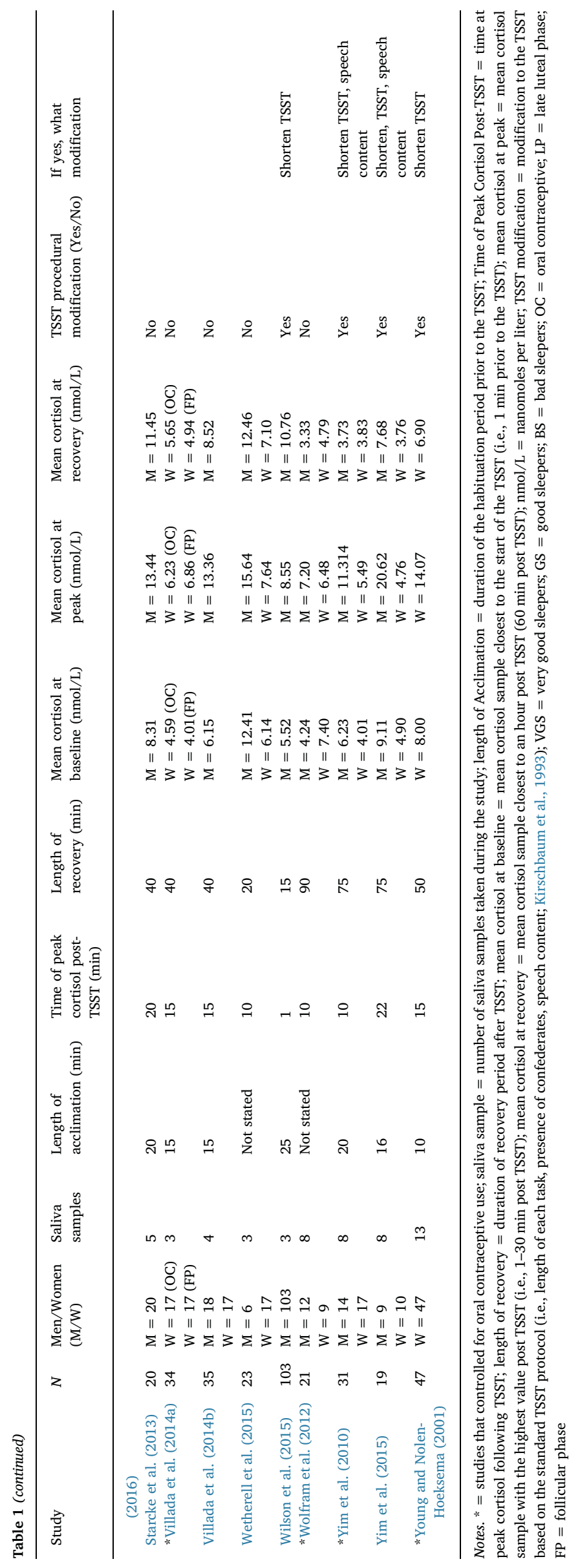




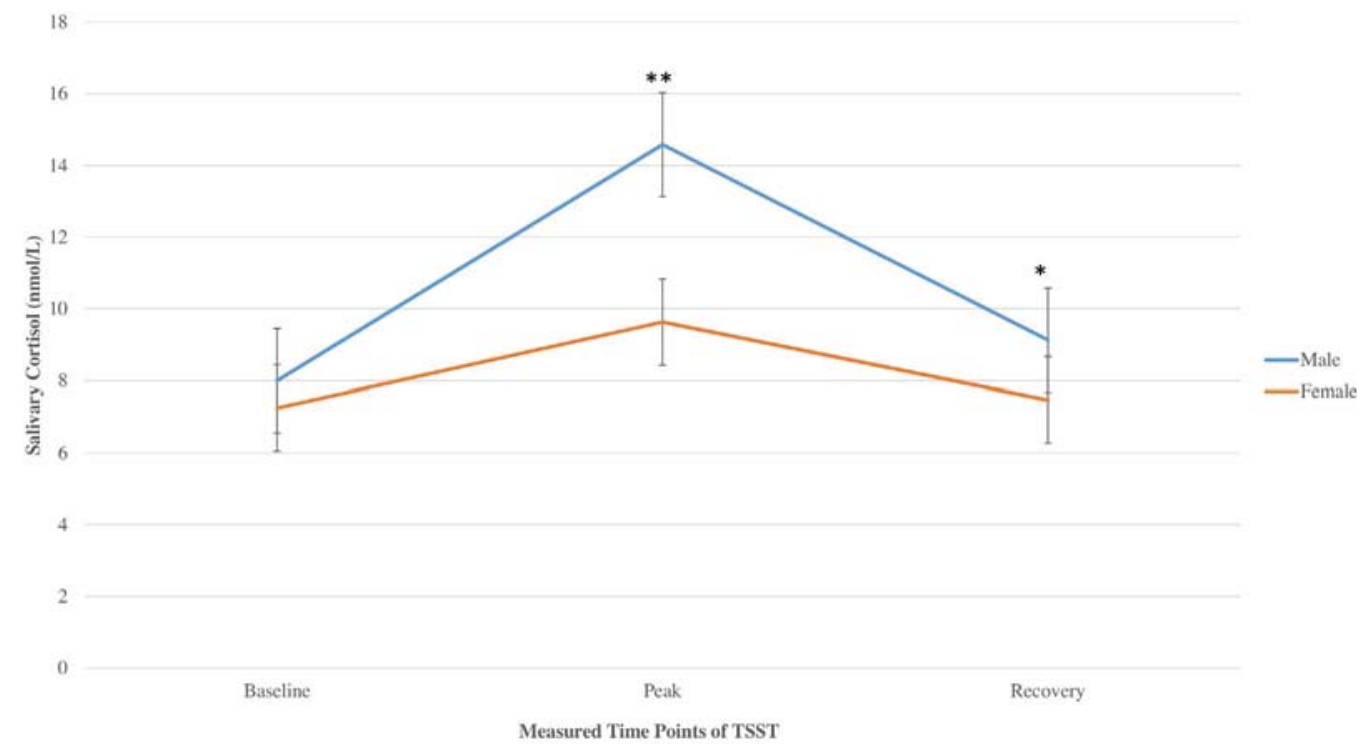

Fig. 2. Sex differences to the TSST in salivary cortisol at sampling time points. ${ }^{* * *} p<.001 ;{ }^{*} p<.01$.

of OC use, we then ran separate analyses comparing men with women on OC, and men with women not on OC. At baseline, tests of homogeneity revealed no differences in salivary cortisol across sexes when comparing men with women using $\mathrm{OC},(Q=2.26, d f=1$, $p=.13)$, or women not using OC $(Q=0.04, d f=1, p=.84)$. At peak, tests of homogeneity revealed a significant effect of sex on salivary cortisol when comparing men with women using $O C,(Q=15.88$, $d f=1, p<.001$ ), while the effects of sex on salivary cortisol approached significance when comparing men with women not using OC ( $Q=3.67, d f=1, p=.055)$, whereby men had higher values than women. Finally, during recovery, tests of homogeneity revealed a significant effect of sex on salivary cortisol when comparing men with women using $\mathrm{OC},(Q=6.04, d f=1, p=.01)$, whereby men had higher values than women. However, salivary cortisol across sexes

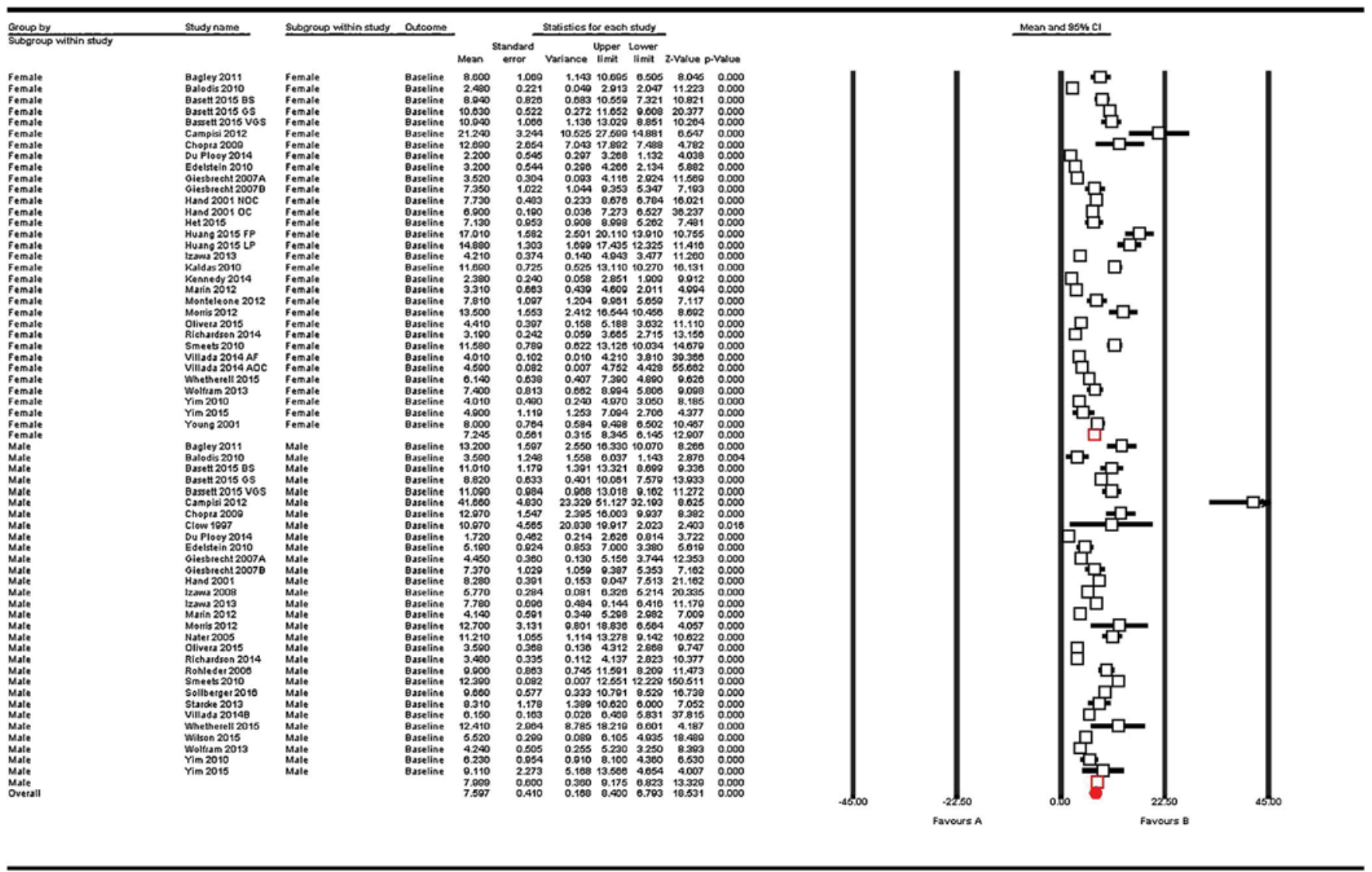

- Square red box indicate sex mean

* Solid red circle indicate overall mean

A. Baseline

Fig. 3. Forrest plots for baseline, peak and recovery. 


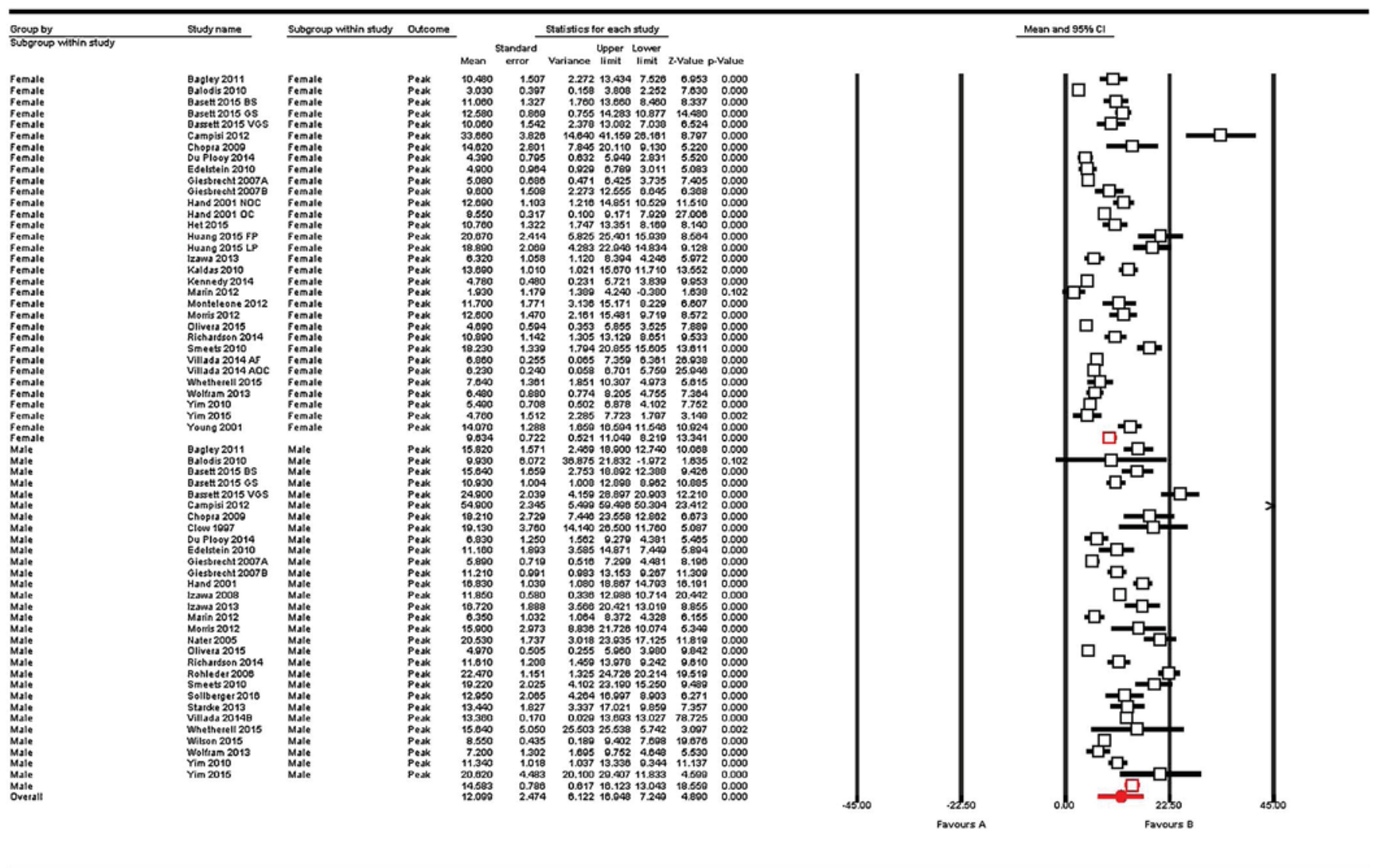

"Square red box indicate sex mean

* Solid red circle indicate overall mean

B. Peak

Fig. 3. (continued)

did not differ when comparing men with women not using OC $(Q=1.81, d f=1, p=.18)$. Summary statistics for these differences are shown in Table 4.

\subsection{Publication bias}

We used Duval and Tweedie's (2000) trim-and-fill procedure to estimate the number of missing studies that might exist. A visual inspection estimation of our funnel plot suggested that our sampled studies were evenly clustered toward the peak, and nested within both sides of the funnel plot. Point estimate of our overall effect sizes for observed and adjusted values were relatively correct, with observed value at $6.68 \mathrm{CI}[6.62,6.74]$, and adjusted value at 5.08 CI [5.03, 5.14]. Fail-safe analysis revealed that it would take 1,322,433 studies with nonsignificant findings to nullify the results of our current metaanalysis. In other words, there would need to be 7110 missing studies for every observed study for the effect to be nullified.

\section{Discussion}

The aims of our current meta-analysis were two-fold: 1) to delineate sex differences in salivary cortisol following the TSST; and 2) to examine the contribution of protocol modifications and procedural variations on sex differences in salivary cortisol responses. At baseline, we did not detect a statistically significant sex difference in salivary cortisol. In contrast, our analyses revealed significant differences at both peak and recovery between the sexes. Further, modification to the TSST protocol did not seem to result in significant differences in salivary cortisol output across studies. Lastly, we found that time of acclimation, peak, and recovery periods moderated salivary cortisol outputs across both sexes.

In our sample of 34 studies, we detected significant heterogeneity in salivary cortisol at peak and recovery. Specifically, men had higher cortisol output at both time points compared to women. According to previous findings, men may interpret stressors differently than women, and exert more effort in response to the confrontations and challenges (Engert et al., 2013; Kirschbaum et al., 1992). Findings from our metaanalysis indicate differences in the trajectory of reactivity between sexes, whereby cortisol levels in men following the TSST were most prominent at peak, and continued through to recovery, relative to cortisol levels in women.

It was evidenced that modification to the standardized TSST protocols (i.e., the length of tasks, content of the speech, and presence of confederates) did not affect differences in salivary cortisol. This suggests that the standardized protocol of this psychosocial stressor is robust despite modifications to its various components.

With respect to our other findings, we found that procedural variations significantly moderated sex differences in salivary cortisol output. Specifically, in studies with an acclimation period of $30 \mathrm{~min}$ or more, sex differences were no longer observed at any points of the reactive cortisol trajectory. In contrast, for studies that had an acclimation period of less than $30 \mathrm{~min}$, we observed differences across all time points. This suggests that the length of acclimation is an important factor that may account for some portion of the sex differences in salivary cortisol across time points, and more specifically, the observable differences in cortisol output between men and women during the anticipatory period. Given the sparse research examining factors that may underlie sex differences in salivary cortisol responses during the TSST, we can only speculate that this relates to previously documented patterns of higher anticipatory salivary cortisol responses 


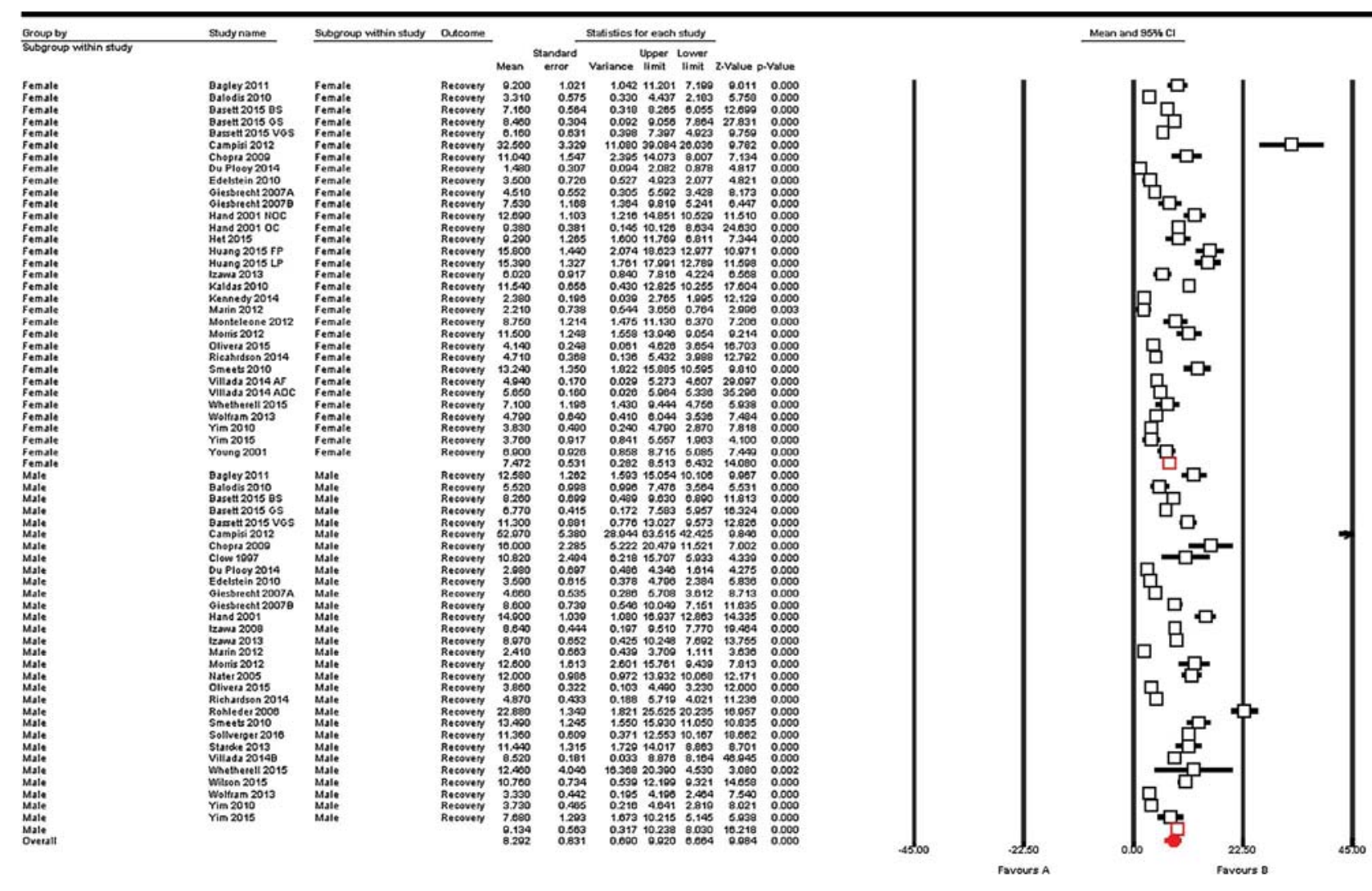

"Square red box indicate sex mean

*** Solid red circle indicate overall mean

C. Recovery

Fig. 3. (continued)

Table 2

Coefficients of model change for meta-regression on procedural variations.

\begin{tabular}{|c|c|c|c|c|c|c|c|c|c|}
\hline & Steps & Slope & $95 \%$ CI & $Z(p)$ & $Q^{b}$ & $d f(Q)$ & $p$ & $\tau^{2}$ & $R^{2}$ \\
\hline \multirow[t]{5}{*}{ Model 1} & Intercept & 7.97 & $5.76,9.84$ & $7.48(<.001)$ & 6641.93 & 145 & $<.001$ & 8.32 & 0.38 \\
\hline & Salivary samples & 0.20 & $-0.13,0.53$ & $1.17(.24)$ & & & & & \\
\hline & Acclimation & 0.05 & $0.01,0.10$ & $2.30(.02)$ & & & & & \\
\hline & Peak & 0.20 & $0.12,0.27$ & $4.82(<.001)$ & & & & & \\
\hline & Recovery & -0.08 & $-0.12,-0.04$ & $-4.24(<.001)$ & & & & & \\
\hline \multirow[t]{4}{*}{ Model 2} & Intercept & 8.41 & $6.61,10.22$ & $9.15(<.001)$ & 7156.80 & 146 & $<.001$ & 8.70 & 0.36 \\
\hline & Acclimation & 0.05 & $0.00,0.09$ & $2.03(.04)$ & & & & & \\
\hline & Peak & 0.19 & $0.11,0.27$ & $4.65(<.001)$ & & & & & \\
\hline & Recovery & -0.07 & $-0.10,-0.04$ & $-4.48(<.001)$ & & & & & \\
\hline
\end{tabular}

Notes. $\tau^{2}$ statistics refers to current model goodness of fit; $Q$ statistics refers to the goodness of fit; analog $R^{2}$.

in men compared to women (Engert et al., 2013; Kirschbaum et al., 1992). Perhaps, a novel laboratory environment contributes to a more pronounced anticipatory response in men than women. Such anticipatory response may act as an additional stressor, wherein it may interact with responses later generated by the TSST.

With regards to timing of peak salivary cortisol, sex differences were found for studies that adhered to the standardized TSST protocol, but not in those that deviated from the protocol. This suggests that salivary cortisol is very time-sensitive, and perhaps there are inherent differences in the trajectory based on times of activation. When sampling peak cortisol, it is important to consider the possibility of differences in the time course of cortisol reactivity in men and women.

In considering the length of recovery, it is important to note that studies with a recovery period of more than 70 min did not find any sex differences at any time points. However, sex differences were evidenced in studies with shorter recovery periods. This suggests that the length of recovery is an important factor that may account for a portion of sex differences in a given study design.

Additionally, it is important to consider that the moderator analyses were conducted independently. Findings revealed that having a longer acclimation period, variability in peak cortisol sampling, and a longer recovery period, all contributed to nullify sex differences in reactive salivary cortisol with regards to the TSST. Taken together, sex differences observed at various time points may be a combination of timing and study design. Notably, we found that length of recovery contributed to sex differences found at peak. Due to the temporal order of the TSST and the lack of theoretical support, it is plausible that experimental design and rigor may underlie this unexpected finding.

Finally, the use of OC significantly impacted variabilities in reactive salivary cortisol. Although women, on average, had lower reactivity than men, NOC women exhibited higher levels of salivary cortisol at peak than those who were on OCs. Although only a small proportion of 
Table 3

$\mathrm{Q}$ statistics on sex differences in salivary cortisol based on procedural variations.

\begin{tabular}{|c|c|c|c|}
\hline & $\begin{array}{l}\text { Baseline }(Q, d f, \\
p)\end{array}$ & Peak $(Q, d f, p)$ & Recovery $(Q, d f, p)$ \\
\hline \multicolumn{4}{|l|}{ Acclimation } \\
\hline$\geq 30 \mathrm{~min}$ & $0.04,1,0.85$ & $2.24,1,0.13^{\mathrm{a}}$ & $2.22,1,0.14^{\mathrm{a}}$ \\
\hline$\leq 29 \mathrm{~min}$ & $5.11,1,0.02^{\mathrm{a}}$ & $28.43,1,<0.001$ & $6.42,1,0.01$ \\
\hline \multicolumn{4}{|l|}{ Peak } \\
\hline $\begin{array}{l}\text { 10-25 min post- } \\
\text { TSST }\end{array}$ & $2.20,1,0.14$ & $21.35,1,<0.001$ & $3.89,1,0.05$ \\
\hline $\begin{array}{l}\leq 9 \mathrm{mins} / \geq 26 \mathrm{~min} \\
\text { Recovery }\end{array}$ & $0.00,1,0.99$ & $0.80,1,0.37^{\mathrm{a}}$ & $0.38,1,0.54^{\mathrm{a}}$ \\
\hline$\geq 70 \mathrm{~min}$ & $0.60,1,0.44$ & $0.88,1,0.35^{\mathrm{a}}$ & $1.28,1,0.26^{\mathrm{a}}$ \\
\hline$\leq 69 \min$ & $2.05,1,0.15$ & $21.37,1,<0.001$ & $8.35,1,<0.01$ \\
\hline
\end{tabular}

Notes. $Q$ statistics refers to homogeneity test; $d f$ refers to degrees of freedom; $p<.05$.

${ }^{\mathrm{a}}$ Indicates moderator effects of procedural variations that are significantly different from initial findings on sex differences.

Table 4

Summary statistics on sex differences in salivary cortisol based on oral contraceptive use.

\begin{tabular}{|c|c|c|c|}
\hline & Mean difference & Standard error $\left(S E_{D}\right)$ & Confidence interval (CI) \\
\hline \multicolumn{4}{|c|}{ Baseline } \\
\hline OC & 2.83 & 0.23 & $\begin{array}{l}\text { Men CI }[6.56,9.51] \\
\text { Women CI }[1.84,8.59]\end{array}$ \\
\hline NOC & 0.34 & 0.11 & $\begin{array}{l}\text { Men CI }[6.69,9.91] \\
\text { Women CI }[5.13,10.79]\end{array}$ \\
\hline \multicolumn{4}{|r|}{ 然 } \\
\hline OC & 8.31 & 0.71 & $\begin{array}{l}\text { Men CI }[13.00,16.32] \\
\text { Women CI }[2.61,10.08]\end{array}$ \\
\hline NOC & 3.68 & 0.29 & $\begin{array}{l}\text { Men CI }[12.89,16.64] \\
\text { Women CI }[7.83,14.35]\end{array}$ \\
\hline \multicolumn{4}{|c|}{ Recovery } \\
\hline OC & 3.77 & 0.32 & $\begin{array}{l}\text { Men CI }[8.03,10.42] \\
\text { Women CI }[2.69,8.22]\end{array}$ \\
\hline NOC & $1.80^{\mathrm{a}}$ & 0.14 & $\begin{array}{l}\text { Men CI }[8.02,10.62] \\
\text { Women CI }[5.24,9.80]\end{array}$ \\
\hline
\end{tabular}

Notes. OC refers to women on oral contraceptives; NOC refers to women not on oral contraceptives; $S E_{D}$ refers to the standard error of mean difference; CI refers to the confidence intervals.

${ }^{\mathrm{a}}$ Indicates moderator effects of procedural variations that are significantly different from initial findings on sex differences.

studies screened and controlled for oral contraceptive use in women, the heterogeneity in values at peak indicate that this is an important factor that contributes to variabilities in reactive cortisol in women. Indeed, past studies documented reduced salivary cortisol responses to stressors, as well as observed differences in diurnal cortisol patterns, in women using oral contraceptives (Roche et al., 2013). It has been suggested that the use of OC may contribute to cortisol binding capacity in women (Durber et al., 1976; Zimmerman et al., 2014). However, it is interesting to note that despite this documented variability in women associated with the use of $\mathrm{OC}$, the peak reactive salivary cortisol remained higher in men. Yet, sampling restrictions placed upon women may contribute to the magnitude of sex differences evidenced in studies with both men and women.

The current meta-analysis should be viewed in light of some limitations and challenges. A limitation is that we were unable to calculate area under the curve (AUC), which is another indicator of cortisol output. This was further amplified due to a lack of access to raw data, variability in sampling time, and number of cortisol samples collected across studies. Additionally, sampling restrictions around the collection of salivary cortisol are also difficult to implement. Within each study, we often lacked information whether potential confounds, such as diet, sleep, body mass index (BMI), ethnicity, and age were controlled for. Any of these factors have the potential to influence salivary cortisol output and reactivity. In addition, many studies either did not include any information about the phase of menstrual cycle (i.e., luteal versus follicular phase) in women, or did not specify the methods used to control for the phase of menstrual cycle. As such, we were limited in both our analyses and interpretation of the possible effects of menstrual cycle on overall sex differences in salivary cortisol to the TSST.

Further, it is plausible that study design and experimental rigor could further contribute to the sex differences that were found in the current meta-analysis. Specifically, studies that followed the standardized protocol closely would also have tighter experimental control placed on their sampled population, thereby accounting for some factors that may contribute to sex differences in cortisol output.

A challenge that we encountered was that very few studies primarily focused on stress reactivity. The TSST is often used as a peripheral tool to induce stress in participants. Yet, the primary focus in many studies is not to assess stress reactivity to the TSST. Rather, the TSST is often incorporated into research designs to test for other elements, such as memory recall, or performance under stress.

Finally, salivary cortisol is an output of the HPA axis, which can be influenced by many interactive systems and hormones within the body, including androgen, testosterone, and estrogen (e.g., Juster et al., 2016). As such, sex could be a proxy for other factors, and these differences in salivary output may be a product of the complex interactions among many systems, and external factors that may impact these systems. Future studies should explore the interaction of the various physiological systems, and sampling restrictions placed upon female populations to further elucidate sex differences in cortisol output.

An important methodological challenge in conducting a metaanalysis is obtaining data from fellow researchers. During the data extraction stage, it is necessary for researchers to contact authors to obtain raw data. Despite advances in technology and research methodologies, acquiring usable data from fellow researchers remains a challenge in the current meta-analysis. As mentioned, we were able to recover data from only 12 of 44 identified articles that needed author contact to obtain usable data. We thus encourage fellow scientists to continue to share their data for research purposes.

Despite these challenges and limitations, the current meta-analysis found significant heterogeneity in men and women in salivary cortisol at peak and recovery following the TSST. Further, we examined procedural variations as potential moderating factors to these sex differences. To our knowledge, this was the first meta-analysis that exclusively focused on sex differences in salivary cortisol in response to the TSST. The main findings were consistent with emerging evidence that examined variations in hormonal outcomes by sex (Juster et al., 2016). The current study also provided insight into the reliability of the TSST despite modifications to the initial protocol.

The current meta-analysis provided significant methodological considerations for future stress research. Particularly, we draw attention to the importance of sampling and procedural variations, and the extent to which they can amplify or negate sex differences in salivary cortisol output in response to an acute stressor. It is therefore important for researchers to account for these factors when conceptualizing study designs and methodologies. Further, future research should continue to elucidate factors that may influence sex differences such as age, hormonal variations, and/or use of medication.

\section{Acknowledgement}

We would like to thank Dr. Leslie Atkinson for his guidance and support throughout this endeavor.

References11*Articles included in the final sample of the metaanalysis review are marked with an asterisk.

*Bagley, S.L., Weaver, T.L., Buchanan, T.W., 2011. Sex differences in physiological and affective responses to stress in remitted depression. Physiol. Behav. 104 (2), 180-186. 
http://dx.doi.org/10.1016/j.physbeh.2011.03.004.

"Balodis, I.M., Wynne-Edwards, K.E., Olmstead, M.C., 2010. The other side of the curve: examining the relationship between pre-stressor physiological responses and stress reactivity. Psychoneuroendocrinology 35 (9), 1363-1373. http://dx.doi.org/10. 1016/j.psyneuen.2010.03.011.

"Bassett, S.M., Lupis, S.B., Gianferante, D., Rohleder, N., Wolf, J.M., 2015. Sleep quality but not sleep quantity effects on cortisol responses to acute psychosocial stress. Stress 18 (6), 638-644. http://dx.doi.org/10.3109/10253890.2015.1087503.

Borenstein, M., Hedges, L.V., Higgins, J.P.T., Rothstein, H.R., 2014. Comprehensive metaanalysis: a computer program from research synthesis (version 3). Biostat Inc, Englewood, NJ.

*Campisi, J., Bravo, Y., Cole, J., Gobeil, K., 2012. Acute psychosocial stress differentially in fluences salivary endocrine and immune measures in undergraduate students. Physiol. Behav. 107 (3), 317-321. http://dx.doi.org/10.1016/j.physbeh.2012.09. 003.

Chopra, K.K., Ravindran, A., Kennedy, S.H., Mackenzie, B., Matthews, S., Anisman, H., Bagby, R.M., Farvolden, P., Levitan, R.D., 2009. Sex differences in hormonal responses to a social stressor in chronic major depression. Psychoneuroendocrinology 34, 1-7. http://dx.doi.org/10.1016/j.psyneuen.2009.03.014.

*Clow, A., Patel, S., Najafi, M., Evans, P.D., Hucklebridge, F., 1997. The cortisol response to psychological challenge is preceded by a transcient rise in endogeneous inhibitor of monoamine oxidase. Life Sci. 61 (5), 567-575.

Del Giudice, M., Ellis, B.J., Shirtcliff, E.A., 2011. The Adaptive Calibration Model of stress responsivity. Neurosci. Biobehav. Rev. 35 (7), 1562-1592. http://dx.doi.org/10. 1016/j.neubiorev.2010.11.007.

Dickerson, S.S., Kemeny, M.E., 2004. Acute stressors and cortisol responses: a theoretical integration and synthesis of laboratory research. Psychol. Bull. 130 (3), 355-391. http://dx.doi.org/10.1037/0033-2909.130.3.355.

*du Plooy, C., Thomas, K.G.F., Henry, M., Human, R., Jacobs, W.J., 2014. The fear-factor stress test: an ethical, non-invasive laboratory method that produces consistent and sustained cortisol responding in men and women. Metab. Brain Dis. 29, 385-394. http://dx.doi.org/10.1007/s11011-014-9484-9.

Durber, S.M., Lawson, J., Daly, J.R., 1976. The effect of oral contraceptives on plasma cortisol and cortisol binding capacity throughout the mentrual cycle in normal women. Br. J. Obstet. Gynaecol. 83 (10), 814-818. Retrived from https://www.ncbi. nlm.nih.gov/pubmed/791349.

Duval, S., Tweedie, R., 2000. Trim and fill: a simple funnel-plot-based method of testing and adjusting for publication bias in meta-analysis. Biometrics 56, 455-463.

*Edelstein, R.S., Yim, I.S., Quas, J.A., 2010. Narcissism predicts heightened cortisol reactivity to a psychosocial stressor in men. J. Res. Personal. 44 (5), 565-572. http:// dx.doi.org/10.1016/j.jrp.2010.06.008.

Engert, V., Efanov, S.I., Duchesne, A., Vogel, S., Corbo, V., Pruessner, J.C., 2013. Differentiating anticipatory from reactive cortisol responses to psychosocial stress. Psychoneuroendocrinology 38 (8), 1328-1337. http://dx.doi.org/10.1016/j. psyneuen.2012.11.018.

Gaffey, A.E., Wirth, M.M., Hoks, R.M., Jahn, A.L., Abercrombie, H.C., 2014. Circulating cortisol levels after exogenous cortisol administration are higher in women using hormonal contraceptives: data from two preliminary studies. Stress 17 (4), 314-320. http://dx.doi.org/10.3109/10253890.2014.919447.

*Giesbrecht, T., Smeets, T., Merckelbach, H., Jelicic, M., 2007. Depersonalization experiences in undergraduates are related to heightened stress cortisol responses. J. Nerv. Ment. Dis. 195 (4), 282-287. http://dx.doi.org/10.1097/01.nmd.0000253822. 60618.60.

*Hand, D.C., 2001. Cardiovascular, endocrine, and affective responses to an acute psychosocial stressor: the impact of objective control, self-efficacy and oral contraceptive medication. (Master's thesis) Retrieved from Proquest Theses and Dissertations (MQ62345).

Hellhammer, D.H., Wust, S., Kudielka, B.M., 2009. Salivary cortisol as a biomarker in stress research. Psychoneuroendocrinology 34, 163-171. http://dx.doi.org/10.1016/ j.psyneuen.2008.10.026.

*Het, S., Vocks, S., Wolf, J.M., Hammelstein, P., Herpertz, S., Wolf, O.T., 2015. Blunted neuroendocrine stress reactivity in young women with eating disorders. J. Psychosom. Res. 78 (3), 260-267. http://dx.doi.org/10.1016/j.jpsychores.2014.11. 001.

"Huang, Y., Zhou, R., Wu, M., Wang, Q., Zhao, Y., 2015. Premenstrual syndrome is associated with blunted cortisol reactivity to the TSST. Stress 18 (2), 160-168. http:// dx.doi.org/10.3109/10253890.2014.999234.

Izawa, S., Sugaya, N., Shirotsuki, K., Yamada, K.C., Ogawa, N., Ouchi, Y., Nagano, Y., Suzuki, K., Nomura, S., 2008. Salivary dehydroepiandrosterone secretion in respons to acute psychosocial stress and its correlations with biological and psychological changes. Biol. Psychol. 79, 294-298. http://dx.doi.org/10.1016/j.biopsycho.2008. 07.003 .

Izawa, S., Sugaya, N., Kimura, K., Ogawa, N., Yamada, K.C., Shirotsuki, K., Mikami, I., Hirata, K., Nagano, Y., Nomura, S., 2013. An increase in salivary interleukin-6 level following acute psychosocial stress and its biological correlates in healthy young adults. Biol. Psychol. 94 (2), 249-254. http://dx.doi.org/10.1016/j.biopsycho.2013. 06.006.

Juster, R., Raymond, C., Desrochers, A.B., Bourdon, O., Durand, N., Wan, N., Tourjman, V., Kouassi, E., Lesage, A., Lupien, S.J., 2016. Sex hormones adjust "sex-specific" reactive and diurnal cortisol profiles. Psychoneuroendocrinology 63, 282-290.

*Kaldas, J., 2010. Perfectionism, dependency, shame and stress within the context of evaluative threat. (Master's thesis) Retrieved from Proquest Theses and Dissertation (MR68244).

Kelly, M.M., Tyrka, A.R., Anderson, G.M., Price, L.H., Carpenter, L.L., 2008. Sex differences in emotional and psysiological responses to the Trier Social Stress Test. J. Behav. Ther. Exp. Psychol. 39 (1), 87-98. http://dx.doi.org/10.1016/j.jbtep.2007.
02.003 .

*Kennedy, P.J., Cryan, J.F., Quigley, E.M.M., Dinan, T.G., Clarke, G., 2014. A sustained hypothalamic-pituitary-adrenal axis response to acute psychosocial stress in irritable bowel syndrome. Psychol. Med. 44, 3123-3134. http://dx.doi.org/10.1017/ S003329171400052X.

Kirschbaum, C., Wust, S., Hellhammer, D., 1992. Consistent sex differences in cortisol responses to psychological stress. Psychosom. Med. 54, 648-657. http://dx.doi.org/ 10.1097/00006842-199211000-00004.

Kirschbaum, C., Pirke, K., Hellhammer, D.H., 1993. The 'Trier Social Stress Test' - a tool for investigating psychobiological stress responses in a laboratory setting. Neuropsychobiology 28, 76-81.

Kudielka, B.M., Buske-Kirschbaum, A., Hellhammer, D.H., Kirschbaum, C., 2004. HPA axis responses to laboratory psychosocial stress in healthy elderly adults, young adults, and children: impact of age and gender. Psychoneuroendocrinology 29, 83-98. http://dx.doi.org/10.1016/S0306-4530(02)00146-4.

Kudielka, B.M., Kirschbaum, C., 2005. Sex differences in HPA axis responses to stress: a review. Biol. Psychol. 69 (1), 113-132. http://dx.doi.org/10.1016/j.biopsycho.2004. 11.009.

Kudielka, B.M., Gierens, A., Hellhammer, D.H., Wust, S., Schlotz, W., 2012. Salivary cortisol in ambulatory assessment - some dos, don'ts, and some open questions. Psychosom. Med. 74, 418-431. http://dx.doi.org/10.1097/PSY.0b013e31825434c7.

*Marin, M., Morin-Major, J., Schramek, T.E., Beaupre, A., Perna, A., Juster, R., Lupien, S.J., 2012. There Is no news like bad news: women are more remembering and stress reactive after reading real negative news than men. PLoS ONE 7 (10), e47189. http:// dx.doi.org/10.1371/journal.pone.0047189.

"Monteleone, P., Tortorella, A., Scognamiglio, P., Serino, I., Monteleone, A.M., Maj, M., 2012. The acute salivary ghrelin response to a psychosocial stress is enhanced in symptomatic patients with bulimia nervosa: a pilot study. Neuropsychobiology 66 , 230-236. http://dx.doi.org/10.1159/000341877.

*Morris, M.C., Rao, U., Garber, J., 2012. Cortisol responses to psychosocial stress predict depression trajectories: social-evaluative threat and prior depressive episodes as moderators. J. Affect. Disord. 143 (1-3), 223-230. http://dx.doi.org/10.1016/j.jad. 2012.05.059.

*Nater, U.M., Rohleder, N., Gaab, J., Berger, S., Jud, A., Kirschbaum, C., Ehlert, U., 2005. Human salivary alpha-amylase reactivity in a psychosocial stress paradigm. Int. J. Psychophysiol. 55, 333-342. http://dx.doi.org/10.1016/j.ijpsycho.2004.09.009.

*Olivera-Figueroa, L.A., Juster, R., Morin-Major, J.K., Marin, M., Lupien, S.J., 2015. A time to be stressed? Time perspectives and cortisol dynamics among healthy adults. Biol. Psychol. 111, 90-99. http://dx.doi.org/10.1016/j.biopsycho.2015.09.002.

Otte, C., Hart, S., Neylan, T.C., Marmar, C.R., Yaffe, K., Mohr, D., 2005. A meta-analysis of cortisol reponse to challenge in human aging: importance of gender.

Psychoneuroendocrinology 30, 80-91. http://dx.doi.org/10.1016/j.psyneuen.2004. 06.002.

Putnam, K.M., Pizzagalli, D.A., Gooding, D.C., Kalin, N.H., Davidson, R.J., 2009. Neural activity and diurnal variation of cortisol: evidence from brain electrical tomography analysis and relevance to anhedonia. Psychophysiology 45 (6), 886-895. http://dx. doi.org/10.1111/j.1469-8986.2008.00697.x.

*Richardson, C.M.E., Rice, K.G., Devine, D.P., 2014. Perfectionism, emotion regulation, and the cortisol stress response. J. Counsel. Psychol. 61 (1), 110-118. http://dx.doi. $\operatorname{org} / 10.1037 / \mathrm{a} 0034446$.

Roche, D.J.O., King, A.C., Cohoon, A.J., Lovallo, W.R., 2013. Hormonal contraceptive use diminishes salivary cortisol response to psychosocial stress and naltrexone in healthy women. Pharmacol. Biochem. Behav. 109, 84-90. http://dx.doi.org/10.1016/j.pbb. 2013.05.007.

Rohatgi, A., 2016 May. Webplotdigitizer: Web based tool to extract data from plots, images and maps. Retrived from http://arohatgi.info/WebPlotDigitizer/citation. html.

*Rohleder, N., Wolf, J.M., Maldonado, E.F., Kirschbaum, C., 2006. The psychosocial stress-induced increase in salivary alpha-amylase is independent of saliva flow rate. Psychophysiology 43, 645-652. http://dx.doi.org/10.1111/j.1469-8986.2006. 00457.x.

*Smeets, T., 2010. Autonomic and hypothalamic-pituitary-adrenal stress resilience: impact of cardiac vagal tone. Biol. Psychol. 84, 290-295. http://dx.doi.org/10.1016/ j.biopsycho.2010.02.015

*Sollberger, S., Bernauer, T., Ehlert, U., 2016. Stress influences environmental donation behavior in men. Psychoneuroendocrinology 63, 311-319.

*Starcke, K., van Holst, R.J., van den Brink, W., Veltman, D.J., Goudriaan, A.E., 2013. Physiological and endocrine reactions to psychosocial stress in alcohol use disorders: duration of abstinence matters. Alchol.: Clin. Exp. Res. 37 (8), 1343-1350. http://dx. doi.org/10.1111/acer.12103.

Taylor, M.K., Larson, G.E., Lauby, M.D.H., Padilla, G.A., Wilson, I.E., Schmied, E.A. Highfill-McRoy, R.M., Morgan III, C.A., 2014. Sex differences in cardiovascular and subjective stress reactions: prospective evidence in a realistic military setting. Stress 17 (1), 70-78. http://dx.doi.org/10.3109/10253890.2013.869208.

*Villada, C., Hidalgo, V., Almela, M., Mastorci, F., Sgoifo, A., Salvador, A., 2014a. Coping with an acute psychosocial challenge: behavioral and physiological responses in young women. PLoS ONE 9 (12), e114640. http://dx.doi.org/10.1371/journal.pone. 0114640.

*Villada, C., Hidalgo, V., Almela, M., Salvador, A., 2014b. Individual differences in the psychobiological response to psychosocial stress (trier social stress test): the relevance of trait anxiety and coping styles. Stress Health 32, 90-99. http://dx.doi. org/10.1002/smi.2582.

*Wetherell, M.A., Lovell, B., Smith, M.A., 2015. The effects of an anticipated challenge on diurnal cortisol secretion. Stress 18 (1), 42-48. http://dx.doi.org/10.3109/ 10253890.2014.993967.

*Wilson, M.C., Zilioli, S., Ponzi, D., Henry, A., Kubicki, K., Nickels, N., Maestripieri, D., 
2015. Cortisol reactivity to psychosocial stress mediates the relationship between extraversion and unrestricted sociosexuality. Personal. Indiv. Diff. 86, 427-431. http://dx.doi.org/10.1016/j.paid.2015.07.003.

*Wolfram, M., Bellingrath, S., Feuerhahn, N., Kudielka, B.M., 2012. Cortisol responses to naturalistic and laboratory stress in student teachers: comparison with a non-stress control day. Stress Health 29, 143-149. http://dx.doi.org/10.1002/smi.2439.

*Yim, I.S., Quas, J.A., Cahill, L., Hayakawa, C.M., 2010. Children's and adults' salivary cortisol responses to an identical psychosocial laboratory stressor.

Psychoneuroendocrinology 35, 241-248. http://dx.doi.org/10.1016/j.psyneuen. 2009.06.014.

*Yim, I.S., Quas, J.A., Rush, E.B., Granger, D.A., Skoluda, N., 2015. Experimental manipulation of the Trier Social Stress Test-Modified (TSST-M) to vary arousal across development. Psychoneuroendocrinology 57, 61-71. http://dx.doi.org/10.1016/j. psyneuen.2015.03.021.

*Young, E.A., Nolen-Hoeksema, S., 2001. Effect of ruminations on the saliva cortisol response to a social stressor. Psychoneuroendocrinology 26, 319-329.

Zimmer, C., Basler, H.D., Vedder, H., Lautenbacher, S., 2003. Sex differences in cortisol responses to noxious stress. Clin. J. Pain 19 (4), 233-239.

Zimmerman, Y., Eijkemans, M.J.C., Coulingh-Bennink, H.J.T., Blankenstin, M.A., Fauser, B.C.J.M., 2014. The effect of combined oral contraception on testosterone levels in healthy women: a systematic review and meta-analysis. Hum. Reprod. Update 20, 76-105. http://dx.doi.org/10.1093/humupd/dmt038. 